
\title{
3 Research Square \\ Clinical observation and prognostic analysis of patients with Klebsiella pneumoniae bloodstream infection
}

shuguang zhang

the first affiliated hospital of zhengzhou university

ziyue yang

the first affiliated hospital of zhengzhou university

limin sun

the first affiliated hospital of zhengzhou university

zhenhua wang

the first affiliated hospital of zhengzhou university

liutao sun

the first affiliated hospital of zhengzhou university;Lankao People's Hospital

jinli xu

the first affiliated hospital of zhengzhou university;Traditional Chinese Medicine Hospital of Sui County

li zeng

the first affiliated hospital of zhengzhou university

tongwen sun ( $\sim$ suntongwen@163.com )

the first affliliated hospital of zhengzhou university https://orcid.org/0000-0001-6536-3222

\section{Research}

Keywords: Klebsiella pneumoniae, Bloodstream infection, Intensive care unit, Anti-Bacterial Agents.

Posted Date: April 23rd, 2020

DOI: https://doi.org/10.21203/rs.3.rs-22419/v1

License: (c) (1) This work is licensed under a Creative Commons Attribution 4.0 International License.

Read Full License 


\section{Abstract}

Background and Purpose: The clinical prognosis of Klebsiella pneumoniae bloodstream infection is poor, and the prevalence of drug-resistant bacteria makes clinical anti-infective treatment more challenging. This retrospective study evaluated the epidemiological characteristics of patients with Klebsiella pneumoniae, the risk factors for drug-resistant bacterial infection and death, and analyzed treatment options.

Methods: Clinical data of 297 patients diagnosed with Klebsiella pneumoniae bacteremia between June 2014 and June 2019 were collected.

Results: Intensive care unit hospitalization history, operation history, recent antibiotic use history, mechanical ventilation, and number of days hospitalized before bloodstream infection were found to be independent risk factors for drug-resistant bacterial infection. The risk of death for carbapenem-resistant Klebsiella pneumoniae infection was 2.942 times higher than that for carbapenem-sensitive Klebsiella pneumoniae infection. For extensively drug-resistant Klebsiella pneumoniae bacteremia patients, the mortality rate of combined anti-infective therapy was lower.

Conclusions: Clinicians should pay attention to patients with high-risk drug-resistant bacteria infection and administer timely anti-infection treatment. The findings of this study may provide some suggestions for early identification and standardized treatment of patients with Klebsiella pneumoniae bacteremia.

\section{Background}

Enterobacteriaceae is a family of pathogens that cause serious infections, among which Escherichia coli and Klebsiella are the most common(1). Klebsiella pneumoniae, a gram-negative bacillus, is the most important class of Klebsiella in the Enterobacteriaceae family(2). These facultative anaerobic bacteria have low nutritional requirements, no flagella, no spores, an obvious capsule, and strong resistance to the outside world. Klebsiella pneumoniae can colonize in the respiratory tract and skin, as well as become aerosolized(3-5). There are a variety of clinical manifestations, including bloodstream infection (BSI), pulmonary infection, urinary tract infection, abdominal infection, and so forth $(6,7)$.

Clinicians pay the most attention to BSI caused by Klebsiella pneumoniae. BSI involves various pathogenic microorganisms, such as bacteria or fungi, invading the blood circulation, reproducing in the blood, releasing toxins and metabolites, and inducing the release of cytokines. This can cause systemic infection, poisoning, and inflammation, which may further lead to lower blood pressure and changes in the blood coagulation and fibrinolytic system. This can cause systemic multiple organ dysfunction syndrome (MODS), a serious systemic infectious disease, and even death. Moreover, BSI can increase the length of hospital stay, treatment costs, and complications after discharge, directly or indirectly affecting patient prognosis. It is reported that the case fatality rate of $\mathrm{BSI}$ is between $21 \%$ and $69 \%(8-11)$. 
Carbapenem antibiotics are powerful $\beta$-lactam drugs for the treatment of Klebsiella pneumoniae-related BSIs. Once resistant to carbapenem antibiotics, clinical treatment of this strain of infection is very difficult. In recent years, the detection rate of carbapenem-resistant Klebsiella pneumoniae (CRKP) has also increased every year(1,12-15). The emergence of CRKP makes the anti-infective treatment encounter great challenges globally(1). A study in Greece found that $10 \%$ of intensive care unit (ICU) patients had CRKP infection, and the mortality rate was four times higher than that of sensitive bacterial infections. However, clinicians are more concerned about extensively drug-resistant (XDR) Klebsiella pneumoniae (XDR-KP), which is resistant to more types of antibiotics than CRKP. XDR-KP is resistant to many antibiotics currently used in the clinic, except for tegacycline and polymyxin. BSI induced by these strains often aggravates the disease, resulting in difficult treatment and poor clinical prognosis. A large number of studies have shown that early identification and early appropriate antibiotic treatment are significantly related to the reduction of mortality (16-20). Due to the lack of attention to high risk factors, late identification and inadequate antibacterial treatment increases mortality; thus, it is necessary to obtain cultures in the early stage of infection to determine specific pathogens, drug resistance, and reasonable antibiotic treatment.

Previous studies have discussed BSIs caused by Klebsiella pneumoniae. However, the risk factors for drug-resistant bacterial infection and death of patients with bacteremia are still inconsistent among the different studies; thus, these risk factors still need to be further improved and verified in clinical practice $(8,21-23)$. Early detection of risk factors and improving clinician awareness play a positive role in the prognosis of patients. Because inadequate anti-infective treatment can increase mortality, improving the identification of patients with high-risk drug-resistant bacteria is key. Once there are clinical symptoms, with respect to drug-resistant bacterial infection, whether combination therapy with antiinfective treatment is effective still needs to be further studied. Some observational studies have shown that, when confirming CRKP infection, combination therapy is related to an improvement of survival rate, but the best treatment scheme has not been determined(24,25). Therefore, we conducted a single-center retrospective study to explore the clinical epidemiology, risk factor analysis, antimicrobial analysis, and prognosis analysis of BSI patients caused by Klebsiella pneumoniae. We analyzed the demographic characteristics of these patients, the risk factors for drug-resistant bacterial infection, and the risk factors for death. Additionally, the treatment schemes in the retrospective data were analyzed. The findings in this study are important for early detection, early treatment, and improving the prognosis of patients with BSI.

\section{Methods}

\subsection{Research objects}

This retrospective observational study was conducted in Grade 3A Hospital, the First Affiliated Hospital of Zhengzhou University in Henan Province, China. Clinical data of patients diagnosed with Klebsiella pneumoniae from June 2014 to June 2019 were collected retrospectively. The inclusion criteria were as follows: 1. Klebsiella pneumoniae was found in at least one blood culture, and the patient met the 
diagnostic criteria for blood flow infection. 2. The patient was positive in multiple cultures, and the first culture was positive at the initial observation time. 3 . The age of the patient was $\geq 18$ years old. 4 . The hospitalized patient had complete clinical data. The exclusion criteria were as follows: 1 . The patient was discharged within 24 hours of hospitalization. 2. The patient had more than two kinds of bacterial BSIs. This study was approved by the Ethics Committee of Scientific Research and Clinical Trials of the First Affiliated Hospital of Zhengzhou University (Code 2020-KY-087).

\subsection{Definitions}

The diagnostic criteria for the BSI patients included in this study can be found in the Centers for Disease Control and Prevention (CDC) guidelines (https://www.cdc.gov/nhsn/PDFs/pscManual/4PSC_CLABScurrent.pdf), including primary BSI, secondary BSI, and Central Line-Associated Bloodstream Infection (CLABI). The diagnostic criteria for septic shock were based on the Sepsis 3.0 guidelines jointly issued by the Society of Critical Care Medicine (SCCM) and the European Society of Intensive Care Medicine (ESCIM) in 2016(26). The ventilator application included non-invasive ventilation and invasive ventilation. Non-invasive ventilation included mask ventilation and excluded high-flow nasal catheter oxygen therapy. Invasive ventilation included orotracheal intubation and tracheotomy connected to a ventilator. Referring to the consensus cosponsored by the European and United States CDC in 2012(27), multidrug resistance (MDR) is defined as insensitivity to three or more types of antibiotics (including drug resistance and intermediation) within the antibacterial spectrum. XDR is defined as insensitivity to almost all types of antibiotics except one or two types of antibiotics (mainly polymyxin and tegacycline), and the determination of class resistance is the same as MDR. Pandrug resistance (PDR) is defined as insensitivity to all types of antimicrobials currently used in the clinic. Combination therapy is defined as a regimen that is treated with two or more antibiotics approved by infectious disease specialists in the course of treatment.

\subsection{Microbiology and drug sensitivity test}

Klebsiella pneumoniae was identified using the VITEK2 system (bioMèrieux, Marcy l'Etoile, France). A direct rapid drug sensitivity test was conducted using the positive blood culture bottle and the disk diffusion method. The results of the antibiotic sensitivity test were described according to the guidelines issued by the European Committee on Antimicrobial Susceptibility Testing (EUCAST).

\subsection{Clinical data collection}

Previous cases were consulted and the data were collected as follows: General patient data (i.e. sex, age, and underlying diseases) were collected. Pre-hospitalization data, including hospitalization history, ICU hospitalization history, operation history, antibiotic use history, as well as glucocorticoid and immunosuppressant use history, were recorded. Past patient history was obtained, such as history of diabetes, hypertension, coronary heart disease, chronic kidney disease (CKD), hematopathy, solid organ tumor, and other recorded diseases. Clinical data before blood culture diagnosis was collected, such as inpatient department, nosocomial infection, Acute Physiology and Chronic Health Evaluation II (APACHE II) score, Sequential Organ Failure Assessment (SOFA) score, Pitt score, Charlson complication index, 
septic shock, use of vasoactive drugs, MODS, respiratory failure and mechanical ventilation, hypoalbuminemia, white blood cell count, and creatinine, myoglobin, glutamic pyruvic transaminase, procalcitonin, and lactic acid levels. Recent invasive procedures were also noted, such as central venous catheter (CVC) placement, temporary dialysis tube placement, arterial puncture, tracheal intubation, tracheotomy, thoracic puncture, abdominal puncture, lumbar puncture, bone marrow puncture, indwelling catheter, and indwelling gastric tube. The results of the blood culture and drug sensitivity tests were recorded. The antibiotic treatment plan and the clinical data of the patients after commencing treatment were recorded. The main outcome index was the 28-day mortality after the diagnosis of BSI, and the secondary outcome indicators included total hospital stay duration, ICU hospital stay duration, mechanical ventilation time, vasoactive drug use, acute renal failure, and MODS.

\subsection{Statistical methods}

The Windows version of SPSS 21.0 software was used for analysis (IBM SPSS Statistics, IBM Corporation, Armonk, New York). Continuous variables with a normal distribution were represented by the mean \pm standard deviation (Mean $\pm S D$ ), and the non-normal distribution data were represented by the median [interquartile range] (Median[IQR]). In this experiment, only the age data had a normal distribution, and the age comparison between the two groups was analyzed using two independent sample t-tests. Data with a non-normal distribution were calculated using the Mann-Whitney $\mathrm{U}$ test. Categorical variables were compared using a $\chi^{2}$ test or Fisher's exact test to compare the differences between groups. Variables with $p<0.05$ upon univariate analysis were included in the multivariate analysis. The risk factors for drug-resistant bacterial infection and the independent predictors of 28-day mortality were determined using binomial logistic regression. The $p$ value, odds ratio (OR), and $95 \%$ confidence interval $(\mathrm{Cl})$ of the risk factors were calculated to evaluate the intensity of the association. A $p$ value less than 0.05 was considered statistically significant.

\section{Results}

This retrospective observational study included 297 patients with Klebsiella pneumoniae BSI. The average age was $55 \pm 16$ years old, of which 202 (68\%) were male patients. Among the positive blood culture results, there were 114 sensitive Klebsiella pneumoniae (S-KP) cases, 99 multidrug-resistant Klebsiella pneumoniae (MDR-KP) cases, and 84 XDR-KP cases. There were no PDR bacteria in these patients.

\subsection{Drug-resistant bacterial infection clinical characteristics and risk factors}

According to the different results from the drug sensitivity test, patients were divided into three groups: the S-KP group, MDR-KP group, and XDR-KP group. The demographics and clinical characteristics of the three groups are shown in Table 1. There was no difference in sex and age between the different groups. By comparing the S-KP group with the drug resistant group (including MDR-KP and XDR-KP groups), we 
found that patients who were hospitalized within 30 days before infection $(p=0.001)$, recent history of ICU hospitalization $(p<0.001)$ and patients who have been operated on recently $(p<0.001)$ were more likely to be infected with drug-resistant bacteria. We compared the recent invasive procedures between the sensitive group and the drug-resistant group and found that the drug resistant group had a more recent indwelling CVC $(p<0.001)$, trachea cannula $(p<0.001)$, tracheotomy $(p=0.001)$, arterial puncture $(p=0.001)$, thoracentesis $(p=0.001)$, indwelling catheter $(p<0.001)$, and gastric tube $(p<0.001)$. Despite the lack of statistical significance, there were more cases of dialysis tube placement in the drug-resistant group, suggesting that such cases require more attention from clinicians. Since fewer patients with a history of organ transplantation, corticosteroid use, or immunosuppressant use were included in the study, the clinical reference value of the comparison between the two groups was small. In the drugresistant bacterial infection group, more patients were treated with antibiotics intravenously $(p=0.045)$. The drug resistant group had a longer hospital stay before the diagnosis of BSI $(p<0.001)$. With respect to the ward in which the patient was admitted at the time of the BSI diagnosis, we found that there were more sensitive BSIs in the internal medicine ward $(p=0.001)$ and more resistant infections in the ICU ( $p=$ 0.002). When comparing basic diseases between the two groups, there were more diabetic patients in the drug-resistant group $(p=0.007)$. We also included the basic information of the patients from the day of the BSI diagnosis. From this information, we can see that the Pitt score $(p<0.001)$, acute APACHE II score $(p<0.001)$, and SOFA score $(p=0.006)$ of the drug-resistant bacteria group were higher. There were more patients with respiratory failure in the drug-resistant bacteria group $(p=0.007)$, more patients under recent ventilation $(p<0.001)$, and more patients with MODS $(p<0.001)$, hypoalbuminemia $(p=0.05)$, and septic shock $(p<0.001)$. 
Table 1

Clinical characteristics of patients in different groups before diagnosis of bloodstream infection

\begin{tabular}{|c|c|c|c|c|c|}
\hline Characteristic & $\begin{array}{l}\text { All } \\
\text { patients }\end{array}$ & S-KP & MDR-KP & XDR-KP & $p^{*}$ \\
\hline $\mathrm{n}$ & 297 & 114 & 99 & 84 & \\
\hline $\operatorname{Gender}^{\phi}($ Male, \%) & $202(68)$ & $73(64)$ & $69(69.6)$ & $60(71.4)$ & 0.246 \\
\hline Age ${ }^{\oiint}$ (Years) & $55 \pm 16$ & $55 \pm 16$ & $56 \pm 16$ & $53 \pm 16$ & 0.798 \\
\hline Hospital before ${ }^{1 \oint}$ & $149(50.1)$ & $43(37.7)$ & $47(47.4)$ & $59(70.2)$ & 0.001 \\
\hline ICU before ${ }^{1 \varnothing}$ & $113(38)$ & $21(18.4)$ & 33 (33.3) & $59(70.2)$ & $\begin{array}{l}< \\
0.001\end{array}$ \\
\hline Surgery $2 \oint$ & $105(35.3)$ & $25(21.9)$ & $35(35.3)$ & $45(53.5)$ & $\begin{array}{l}<.001 \\
0.001\end{array}$ \\
\hline \multicolumn{6}{|l|}{ Invasive operation ${ }^{3 \phi}$} \\
\hline CVC & $144(48.4)$ & $35(30.7)$ & $49(49.4)$ & $60(71.4)$ & $\begin{array}{l}<.001 \\
0.001\end{array}$ \\
\hline Subclavian vein & $89(61.8)$ & $26(74.2)$ & $25(51)$ & 38 (63.3) & 0.081 \\
\hline Internal jugular vein & $46(31.9)$ & $7(20)$ & $21(42.8)$ & $18(30)$ & 0.082 \\
\hline Femoral vein & $9(6.2)$ & $2(5.7)$ & $3(6.1)$ & $4(6.6)$ & 1 \\
\hline Dialysis tube & $46(15.4)$ & $12(10.5)$ & $14(14.1)$ & $20(23.8)$ & 0.062 \\
\hline Arteriopuncture & $183(61.6)$ & $57(50)$ & $55(55.5)$ & $71(84.5)$ & 0.001 \\
\hline Trachea cannula & $87(29.2)$ & $15(13.1)$ & $34(34.3)$ & $38(45.2)$ & $\begin{array}{l}<.001 \\
0.001\end{array}$ \\
\hline Tracheotomy & $32(10.7)$ & $4(3.5)$ & $14(14.1)$ & $14(16.6)$ & 0.001 \\
\hline Thoracentesis & $16(5.3)$ & 0 & $11(11.1)$ & $5(5.9)$ & 0.001 \\
\hline Abdominocentesis & $18(6)$ & $6(5.2)$ & $7(7.1)$ & $5(5.9)$ & 0.649 \\
\hline Lumbar puncture & $23(7.7)$ & $6(5.2)$ & $9(9.1)$ & $8(9.5)$ & 0.207 \\
\hline Bone marrow aspiration & $35(11.7)$ & $13(11.4)$ & $10(10.1)$ & $12(14.2)$ & 0.872 \\
\hline
\end{tabular}

*Single factor comparison: The comparison of sensitivity $(n=114)$ and drug resistance $(n=183)$, in which drug resistance includes MDR-KP and XDR-KP. Chi-Square Test is used to calculate the twocategory variables, t-test is used to calculate the normal distribution variables, and Mann-Whitney $\mathrm{U}$ Test is used to calculate the variables with non-normal distribution and uneven variance.

Continued Table 1. Clinical characteristics of patients in different groups before diagnosis of bloodstream infection 


\begin{tabular}{|c|c|c|c|c|c|}
\hline Characteristic & $\begin{array}{l}\text { All } \\
\text { patients }\end{array}$ & S-KP & MDR-KP & XDR-KP & $p^{*}$ \\
\hline Indwelling catheter & $168(56.5)$ & $45(39.4)$ & $56(56.5)$ & $67(79.7)$ & $<.001$ \\
\hline Indwelling gastric tube & $120(40.4)$ & $30(26.3)$ & $36(36.3)$ & $54(64.2)$ & <. \\
\hline Organ transplantation $4 \oint$ & $9(3)$ & $2(1.7)$ & $1(1)$ & $6(7.1)$ & 0.311 \\
\hline Application of antibiotics ${ }^{1 \oint}$ & $96(32.3)$ & $29(25.4)$ & $36(36.3)$ & $31(36.9)$ & 0.045 \\
\hline Glucocorticoid $^{1} \oint$ & $18(6)$ & $4(3.5)$ & $8(8.1)$ & $6(7.1)$ & 0.146 \\
\hline Immunosuppressant ${ }^{1 \varnothing}$ & $22(7.4)$ & $7(6.1)$ & $9(9.1)$ & $6(7.1)$ & 0.510 \\
\hline Hospital infection ${ }^{\phi}$ & $162(54.5)$ & $58(50.8)$ & $54(54.5)$ & $50(59.5)$ & 0.316 \\
\hline $\begin{array}{l}\text { Hospital days before } \\
\text { infection } \oiint\end{array}$ & $10[4,20]$ & $6[3,11]$ & $11[5,23]$ & $16[9,24]$ & $<.001$ \\
\hline Characteristic & $\begin{array}{l}\text { All } \\
\text { patients }\end{array}$ & S-KP & MDR-KP & XDR-KP & \\
\hline \multicolumn{6}{|l|}{ Inpatient department ${ }^{5}$} \\
\hline Internal Medicine & $106(35.6)$ & $54(47.3)$ & $34(34.3)$ & $18(21.4)$ & 0.001 \\
\hline Surgery Ward & $64(21.5)$ & $24(21)$ & $26(26.2)$ & $14(16.6)$ & 0.87 \\
\hline ICU & $127(42.7)$ & $36(31.5)$ & $39(39.3)$ & $52(61.9)$ & 0.002 \\
\hline Pitt bacteremia score $\oiiint$ & $2[0,4]$ & $1[0,2]$ & $2[0,5]$ & $3[1,6]$ & $<.001$ \\
\hline Charlson comorbidity index $\oiint$ & $3[2,4]$ & $3[2,5]$ & $3[2,4]$ & $2[1,4]$ & 0.443 \\
\hline \multicolumn{6}{|l|}{ Basic diseases } \\
\hline Hypertension & $86(28.9)$ & $31(27.1)$ & $26(26.2)$ & $29(34.5)$ & 0.579 \\
\hline Diabetes & $69(23.2)$ & $17(14.9)$ & $25(25.2)$ & $27(32.1)$ & 0.007 \\
\hline Chronic renal failure & $47(15.8)$ & $19(16.6)$ & $10(10.1)$ & $18(21.4)$ & 0.754 \\
\hline Liver insufficiency & $47(15.8)$ & $17(14.9)$ & $14(14.1)$ & $16(19)$ & 0.734 \\
\hline \multicolumn{6}{|c|}{$\begin{array}{l}\text { * Single factor comparison: The comparison of sensitivity }(n=114) \text { and drug resistance }(n=183) \text {, in } \\
\text { which drug resistance includes MDR-KP and XDR-KP. Chi-Square Test is used to calculate the two- } \\
\text { category variables, t-test is used to calculate the normal distribution variables, and Mann-Whitney U } \\
\text { Test is used to calculate the variables with non-normal distribution and uneven variance. }\end{array}$} \\
\hline $\begin{array}{l}\text { Continued Table 1. Clinical cha } \\
\text { bloodstream infection }\end{array}$ & teristics of & tients in $\mathrm{d}$ & nt groups & e diagno & \\
\hline
\end{tabular}




\begin{tabular}{|c|c|c|c|c|c|}
\hline Characteristic & $\begin{array}{l}\text { All } \\
\text { patients }\end{array}$ & S-KP & MDR-KP & XDR-KP & $p^{*}$ \\
\hline Solid Organ Cancer & $36(12.1)$ & $14(12.2)$ & $14(14.1)$ & $8(9.5)$ & 0.947 \\
\hline Blood disease & $40(13.4)$ & $16(14)$ & $11(11.1)$ & $13(15.4)$ & 0.821 \\
\hline Coronary heart disease & $58(19.5)$ & $21(18.4)$ & $16(16.1)$ & $21(25)$ & 0.704 \\
\hline Respiratory failure ${ }^{\oint}$ & $60(20.2)$ & $14(12.2)$ & $13(13.1)$ & $33(39.2)$ & 0.007 \\
\hline Hypoalbuminemia $\oint^{\prime}$ & $90(30.3)$ & $27(23.6)$ & 31 (31.3) & $32(38)$ & 0.05 \\
\hline White blood cell $\oiint$ & $\begin{array}{l}9 \\
{[5.5,14.4]}\end{array}$ & $\begin{array}{l}8.2 \\
{[4.8,13.5]}\end{array}$ & $\begin{array}{l}8.2 \\
{[5.5,14.1]}\end{array}$ & $\begin{array}{l}10.5 \\
{[6.3,16.4]}\end{array}$ & 0.339 \\
\hline Alanine aminotrans $\oiint$ & $30[16,61]$ & $33[17,60]$ & $30[17,59]$ & $27[16,66]$ & 0.329 \\
\hline Creatinine $\oiint$ & $\begin{array}{l}69 \\
{[49,106]}\end{array}$ & $69[50,90]$ & $62[48,95]$ & $85[53,171]$ & 0.94 \\
\hline Septic shock ${ }^{\oint}$ & $63(21.2)$ & $11(9.6)$ & $20(20.2)$ & $32(38.1)$ & $<.001$ \\
\hline MODS ${ }^{\phi}$ & $48(16.1)$ & $7(6.1)$ & $14(14.1)$ & $27(32.1)$ & $<.001$ \\
\hline APACHE\score & $11[6,18]$ & $8[5,14]$ & $12[7,17]$ & $17[9,22]$ & $\begin{array}{l}<.001 \\
0 .\end{array}$ \\
\hline SOFA score $\oiint$ & $4[2,7]$ & $3[2,6]$ & $4[2,7]$ & $7[3,10]$ & 0.006 \\
\hline Mechanical ventilation ${ }^{\phi}$ & $95(31.9)$ & $12(10.5)$ & $36(36.3)$ & $47(55.9)$ & $\begin{array}{l}< \\
0.001\end{array}$ \\
\hline \multicolumn{6}{|c|}{$\begin{array}{l}{ }^{1} 30 \text { days before this hospitalization; }{ }^{2} 90 \text { days before this hospitalization; }{ }^{3} 30 \text { days before positive } \\
\text { blood culture; }{ }^{4} \text { Before this hospitalization (no matter how long ago); }{ }^{5} \text { The in-patient department on } \\
\text { the day of blood sampling and examination; }{ }^{\oint} \mathrm{n}(\mathrm{n} \%) ;{ }^{\$} \text { Mean } \pm \text { SD; }{ }^{\Uparrow} \text { Median[IQR] }\end{array}$} \\
\hline \multicolumn{6}{|c|}{$\begin{array}{l}\text { Abbreviation: } n \text { sample size, } S-K P \text { sensitive Klebsiella pneumoniae, } M D R-K P \text { multidrug resistant } \\
\text { Klebsiella pneumoniae, } X D R-K P \text { extensively drug resistant Klebsiella pneumoniae, ICU intensive care } \\
\text { unit, } C V C \text { central venous catheter, MODS multiple organ dysfunction syndrome, } A P A C H E \text { acute } \\
\text { physiology and chronic health evaluation, SOFA sequential organ failure assessment. }\end{array}$} \\
\hline \multicolumn{6}{|c|}{$\begin{array}{l}\text { *Single factor comparison: The comparison of sensitivity }(n=114) \text { and drug resistance }(n=183) \text {, in } \\
\text { which drug resistance includes MDR-KP and XDR-KP. Chi-Square Test is used to calculate the two- } \\
\text { category variables, t-test is used to calculate the normal distribution variables, and Mann-Whitney } U \\
\text { Test is used to calculate the variables with non-normal distribution and uneven variance. }\end{array}$} \\
\hline \multicolumn{6}{|c|}{$\begin{array}{l}\text { Continued Table 1. Clinical characteristics of patients in different groups before diagnosis of } \\
\text { bloodstream infection }\end{array}$} \\
\hline
\end{tabular}

Univariate analysis showed that recent hospitalization history, ICU hospitalization history, operation history, invasive operation history, antibiotic application history, hospitalization time, the hospitalization 
ward, scores, respiratory failure, MODS, and septic shock were the risk factors for Klebsiella pneumoniae $\mathrm{BSI}$. After adjusting the hospitalization time and scores using binary logistic regression, we found that ICU hospitalization history $(\mathrm{OR}=2.32,95 \% \mathrm{Cl} 1.22-4.4, p=0.01)$, surgical history $(\mathrm{OR}=2.33,95 \% \mathrm{Cl}$ $1.26-4.32, p=0.007)$, recent antibiotic use history ( $\mathrm{OR}=2.02,95 \% \mathrm{Cl} 1.1-3.74, p=0.024)$, mechanical ventilation $(\mathrm{OR}=3.3,95 \% \mathrm{Cl} 1.56-6.97, p=0.002)$, and days of hospitalization before $\mathrm{BSI}$ were independent risk factors for drug-resistant bacterial infection. The results of the multivariate analysis are shown in Table 2. By dividing the hospitalization time before infection into three groups ( 1 week, 2 weeks, and more than 2 weeks), we found that patients with more than 2 weeks of hospitalization before BSI were $4.34(2.21-8.55)$ times more likely to be infected with drug-resistant bacteria than those who were hospitalized within one week. 
Table 2

Logistic regression analysis of risk factors of drug-resistant KP bloodstream infections

\section{Characteristics}

History of ICU hospitalization

None

Have

History of surgery

None

Have

History of application of antibiotics

None

Have

Mechanical ventilation

None

Have

APACHE II score

$\leq 10$

$>10$

Hospitalization days before bloodstream infection

$\leq 7$ days

$>7$ days and $\leq 14$ days

$>14$ days

Abbreviation: $n$ sample size, $K P$ Klebsiella pneumoniae, $O R$ odds ratio, $C /$ Confidence interval, ICU intensive care unit, APACHE Acute Physiology and Chronic Health Evaluation.
OR $(95 \% \mathrm{Cl})$

$p$
$2.32(1.22-4.4) \quad 0.01$

1

$2.33(1.26-4.32) \quad 0.007$

1

$2.02(1.1-3.74) \quad 0.024$

1

$3.3(1.56-6.97) \quad 0.002$

1

$1.9(1.06-3.42)$

0.031

1

$1.77(0.88-3.55) \quad 0.11$

$4.34(2.21-8.55) \quad<0.001$

\subsection{Risk factors for death in patients with Klebsiella pneumoniae BSI}

Taking death within 28 days after BSI diagnosis as the main end point, the results showed that 127 cases died and 170 cases survived. The 28-day mortality rate of patients with Klebsiella pneumoniae BSI was 42.8\%. We compared patients with different clinical outcomes, and the results obtained by univariate analysis are shown in Table 3. As with the risk factors of drug-resistant bacterial infection, recent hospitalization history $(p=0.029)$, ICU hospitalization history $(p<0.001)$, and surgical history $(p=0.026)$ 
occurred more frequently in the death group. The comparison of invasive operation between the survival group and the death group showed that patients with an indwelling CVC $(p<0.001)$, an indwelling dialysis tube $(p<0.001)$, arterial puncture $(p<0.001)$, trachea cannula $(p<0.001)$, tracheotomy $(p=0.022)$, thoracentesis $(p=0.007)$, an indwelling catheter $(p=0.001)$, and a gastric tube $(p<0.001)$ had a higher risk of death. When comparing previous basic diseases between the two groups, there were more patients with diabetes $(p<0.001)$, chronic renal failure $(p=0.004)$, and coronary heart disease $(p=0.006)$ in the death group. From the comparison of clinical characteristics before the BSI diagnosis, patients with respiratory failure $(p=0.001)$, ventilator use $(p<0.001)$, hypoalbuminemia $(p<0.001)$, septic shock $(p<$ $0.001)$, and MODS $(p<0.001)$ predicted a worse clinical outcome. At the same time, we observed that the APACHE II score $(p<0.001)$ and SOFA score $(p<0.001)$ were higher in the death group. In the death group, the hospitalization days before BSI were relatively longer $(8[4,18]$ days versus $14[5,22]$ days, $p=0.015)$. 
Table 3

Comparison of the basic condition of patients between 28-day survivors and non-survivors ( $\mathrm{n}, \%)$

\begin{tabular}{|c|c|c|c|}
\hline Characteristics & $\begin{array}{l}\text { Survivors }(\mathrm{n}= \\
170)\end{array}$ & $\begin{array}{l}\text { Non-survivors }(\mathrm{n}= \\
127)\end{array}$ & $p$ \\
\hline Gender (male, \%) ${ }^{\int}$ & $116(68.2)$ & $86(67.7)$ & 0.924 \\
\hline Age $(\text { Years, Mean } \pm \text { SD })^{\int S}$ & $54 \pm 16$ & $55 \pm 15$ & 0.625 \\
\hline Hospital before ${ }^{\int}$ & $76(44.7)$ & $73(57.4)$ & 0.029 \\
\hline ICU before ${ }^{\int}$ & $39(22.9)$ & $74(58.2)$ & $<.001$ \\
\hline Recent surgical history $\int$ & $51(30)$ & $54(42.5)$ & 0.026 \\
\hline \multicolumn{4}{|l|}{ Invasive operation $\int$} \\
\hline CVC & $57(33.5)$ & $87(68.5)$ & $<$. \\
\hline Subclavian vein & $35(61.4)$ & $54(62.1)$ & 0.936 \\
\hline Internal jugular vein & $18(31.6)$ & $28(32.2)$ & 0.939 \\
\hline Femoral vein & $3(5.3)$ & $6(6.9)$ & 0.692 \\
\hline Dialysis tube & $14(8.2)$ & $32(25.2)$ & $<$. \\
\hline Arteriopuncture & $89(52.4)$ & $94(74)$ & $\dot{0.001}$ \\
\hline Trachea cannula & $21(12.3)$ & $66(51.9)$ & $\dot{0} 001$ \\
\hline Tracheotomy & $9(5.2)$ & $23(18.1)$ & 0.022 \\
\hline Thoracentesis & $4(2.3)$ & $12(9.4)$ & 0.007 \\
\hline Abdominocentesis & $8(4.7)$ & $10(7.9)$ & 0.258 \\
\hline Lumbar puncture & $10(5.9)$ & $13(10.2)$ & 0.165 \\
\hline Bone marrow aspiration & $18(10.6)$ & $17(13.4)$ & 0.459 \\
\hline Indwelling catheter & $82(48.2)$ & $86(67.7)$ & 0.001 \\
\hline Indwelling gastric tube & $48(28.2)$ & $72(56.7)$ & $\dot{0.001}$ \\
\hline Organ transplantation $\int$ & $4(2.3)$ & $5(3.9)$ & 0.656 \\
\hline
\end{tabular}

Continued Table 3. Comparison of the basic condition of patients between 28-day survivors and nonsurvivors $(n, \%)$ 


\begin{tabular}{|c|c|c|c|}
\hline Characteristics & $\begin{array}{l}\text { Survivors }(n= \\
170)\end{array}$ & $\begin{array}{l}\text { Non-survivors }(n= \\
127)\end{array}$ & $p$ \\
\hline Application of antibiotics $\int$ & $59(34.4)$ & $37(29.1)$ & 0.310 \\
\hline Glucocorticoid $\int$ & $9(5.2)$ & $9(7.1)$ & 0.522 \\
\hline Immunosuppressant $\delta$ & $12(7.1)$ & $10(7.8)$ & 0.791 \\
\hline Hospital infection $\int$ & $94(55.2)$ & $63(53.5)$ & 0.764 \\
\hline Hospital days before infection $\iiint$ & $8[4,18]$ & $14[5,22]$ & 0.015 \\
\hline Pitt bacteremia score (median [IQR]) $\iiint$ & $2[0,4]$ & $2[0,5]$ & 0.577 \\
\hline $\begin{array}{l}\text { Charlson comorbidity index (median } \\
{\left[\text { [IQR]) } \iiint^{-}\right.}\end{array}$ & $3[2,5]$ & $3[1,4]$ & 0.277 \\
\hline Characteristics & $\begin{array}{l}\text { Survivors }(n= \\
170)\end{array}$ & $\begin{array}{l}\text { Non-survivors }(n= \\
127)\end{array}$ & $p$ \\
\hline \multicolumn{4}{|l|}{ Basic diseases $\int$} \\
\hline Hypertension & $47(27.6)$ & $39(30.7)$ & 0.565 \\
\hline Diabetes & $26(15.2)$ & $43(33.8)$ & $<.001$ \\
\hline Chronic renal failure & $18(10.5)$ & $29(22.8)$ & 0.004 \\
\hline Liver insufficiency & $21(12.3)$ & $26(20.4)$ & 0.058 \\
\hline Solid Organ Cancer & $23(13.5)$ & $13(10.2)$ & 0.390 \\
\hline Blood disease & $20(11.7)$ & $20(15.7)$ & 0.320 \\
\hline Coronary heart disease & $24(14.1)$ & $34(26.7)$ & 0.006 \\
\hline Respiratory failure $\int$ & $23(13.5)$ & $37(29.1)$ & 0.001 \\
\hline Hypoalbuminemia ${ }^{f}$ & $34(20)$ & $56(44)$ & $<.001$ \\
\hline Drug resistance $(\mathrm{S}-\mathrm{KP}, \%)^{\int}$ & $88(51.7)$ & $26(20.4)$ & $<.001$ \\
\hline Drug resistance (MDR-KP, \%) ${ }^{\int}$ & $56(32.9)$ & $43(33.8)$ & 0.868 \\
\hline Drug resistance $(\mathrm{XDR}-\mathrm{KP}, \%)^{\int}$ & $26(15.2)$ & $58(45.6)$ & $\begin{array}{l}< \\
0.001\end{array}$ \\
\hline
\end{tabular}

Continued Table 3. Comparison of the basic condition of patients between 28-day survivors and nonsurvivors $(n, \%)$ 


\begin{tabular}{|c|c|c|c|}
\hline Characteristics & $\begin{array}{l}\text { Survivors }(n= \\
170)\end{array}$ & $\begin{array}{l}\text { Non-survivors }(n= \\
127)\end{array}$ & $p$ \\
\hline Septic shock $\int$ & $15(8.8)$ & $48(37.7)$ & $<001$ \\
\hline MODS ${ }^{f}$ & $9(5.2)$ & $39(30.7)$ & $<.001$ \\
\hline APACHE\score $\iint S$ & $7[4,11]$ & $18[13,23]$ & $<.001$ \\
\hline SOFA score $\iiint$ & $2[2,4]$ & $8[5,11]$ & $<.001$ \\
\hline Mechanical ventilation $\int$ & $23(13.5)$ & $72(56.6)$ & $<001$ \\
\hline \multicolumn{4}{|c|}{$\begin{array}{l}\int \text { Use Chi-Square Test to verify; } \iint \text { Two groups were compared with independent t-test; } \iiint_{\text {Use Mann- }} \\
\text { Whitney U Test to verify. }\end{array}$} \\
\hline \multicolumn{4}{|c|}{$\begin{array}{l}\text { Abbreviation: SD Standard deviation, ICU intensive care unit, } C V C \text { central venous catheter, IQR inter- } \\
\text { quartile range, MODS multiple organ dysfunction syndrome, } A P A C H E \text { acute physiology and chronic } \\
\text { health evaluation, SOFA sequential organ failure assessment, } S-K P \text { sensitive Klebsiella pneumoniae, } \\
\text { MDR-KP multidrug resistant Klebsiella pneumoniae, XDR-KP extensively drug resistant Klebsiella } \\
\text { pneumoniae. }\end{array}$} \\
\hline \multicolumn{4}{|c|}{$\begin{array}{l}\text { Continued Table } 3 \text {. Comparison of the basic condition of patients between } 28 \text {-day survivors and non- } \\
\text { survivors }(n, \%)\end{array}$} \\
\hline
\end{tabular}

We compared the results of the drug sensitivity test between the survival group and the death group, and the proportion of S-KP patients in the survival group was higher, while the proportion of XDR-KP patients in the death group was higher. There was no significant difference in the distribution of MDR-KP infections between the two groups. The drug-resistance of patients in the survival group and the death group were compared one by one, as shown in Table 4. It was observed that the proportion of drug resistance to penicillins, cephalosporins, and carbapenems in the death group was statistically significantly higher than that in the survival group. According to the clinical data from the day of BSI diagnosis to before discharge, the risk of accident was higher in the 28-day death group. Patients requiring ventilator support, continuous renal replacement therapy (CRRT), MODS, acute renal failure, transfer to ICU, and uncleared bacteria after blood culture diagnosis predicted a poor prognosis (see Table 5). 
Table 4

Comparison of drug resistance between 28-day survivors and non-survivors ( $\mathrm{n}, \%)$

\begin{tabular}{|llll|}
\hline Antibacterial drugs & Survivors $(\mathbf{n}=\mathbf{1 7 0})$ & Non-survivors $(\mathbf{n}=\mathbf{1 2 7})$ & $\boldsymbol{p}$ \\
\hline Piperacillin/tazobactam & $46(27)(3$ intermediates $)$ & $84(66.1)(2$ intermediates $)$ & $<0.001$ \\
\hline Cefoperazone/sulbactam & $81(47.6)(3$ intermediates $)$ & $101(79.5)(2$ intermediates $)$ & $<0.001$ \\
\hline Cefazoline & $93(54.7)$ & $105(82.6)$ & $<0.001$ \\
\hline Ceftriaxone & $82(48.2)(2$ intermediates $)$ & $102(80.3)$ & $<0.001$ \\
\hline Ceftaxime & $64(37.6)(2$ intermediates $)$ & $86(67.7)(1$ intermediate $)$ & $<0.001$ \\
\hline Cefepime & $58(34.1)(1$ intermediate $)$ & $81(63.7)$ & $<0.001$ \\
\hline Amintronem & $70(41.1)$ & $89(70)(3$ intermediates $)$ & $<0.001$ \\
\hline Imipenem & $43(25.2)(1$ intermediate $)$ & $79(62.2)$ & $<0.001$ \\
\hline Ertapenem & $42(24.7)(2$ intermediates $)$ & $82(64.5)$ & $<0.001$ \\
\hline Tobramycin & $50(29.4)(19$ intermediates $)$ & $68(53.5)(17$ intermediates $)$ & $<0.001$ \\
\hline Aamikacin & $35(20.5)$ & $63(49.6)(1$ intermediate $)$ & $<0.001$ \\
\hline Levofloxacin & $59(34.7)(3$ intermediates $)$ & $83(65.3)(4$ intermediates $)$ & $<0.001$ \\
\hline Ciprofloxacin & $65(38.2)(3$ intermediates $)$ & $89(70)(5$ intermediates $)$ & $<0.001$ \\
\hline Cotrimoxazole & $73(42.9)$ & $72(56.6)$ & $<0.001$ \\
\hline Polymyxin B & $0(1$ intermediate $)$ & $0(3$ intermediates $)$ & \\
\hline Tegacycline & $0(2$ intermediates $)$ & $0(5$ intermediates $)$ & \\
\hline
\end{tabular}


Table 5

Comparison of the clinical data between 28-day survivors and non-survivors $(n, \%)$

\begin{tabular}{|c|c|c|c|}
\hline Characteristics & Survivors $(n=170)$ & Non-survivors $(n=127)$ & $p$ \\
\hline Mechanical ventilation $\int$ & $54(31.7)$ & $72(56.6)$ & $<0.001$ \\
\hline Mechanical ventilation time $\iiint$ & $75[29,189]$ & $88[36,178]$ & 0.390 \\
\hline$A K I S$ & $23(13.5)$ & $85(66.9)$ & $<0.001$ \\
\hline CRRT $\int$ & $11(6.4)$ & $30(23.6)$ & $<0.001$ \\
\hline Vasoactive agent $\delta$ & $68(40 \%)$ & $77(60.6)$ & $<0.001$ \\
\hline Hospitalization days $\iiint$ & $23[14,37]$ & $22[11,36]$ & 0.340 \\
\hline Transferred to ICU $\int$ & 88 (51.7) & 95 (74.8) & $<0.001$ \\
\hline ICU hospitalization days $\iiint$ & $14[7,25]$ & $12[6,19]$ & 0.073 \\
\hline bacterial clearance ${ }^{\int}$ & $113(66.4)$ & $42(33)$ & $<0.001$ \\
\hline
\end{tabular}

In this study, binomial logistic regression was used to evaluate the impact of the above suspicious risk factors on the 28-day survival rate of the subjects. The studentized residual of seven patients was more than three times the standard deviation, but remained in the analysis. The final logistic model was statistically significant, with a $\chi^{2}=63.772$ and $p<0.001$. The model can correctly classify $79.5 \%$ of the research objects. The sensitivity, specificity, positive predictive value, and negative predictive value of the model were $69.3 \%, 87.1 \%, 80 \%$, and $79.1 \%$, respectively. Among the independent variables included in the model, tracheal intubation, respiratory failure, hypoalbuminemia, MODS, a SOFA score greater than 6 , and CRKP were statistically significant. The specific information is shown in Table 6. Endotracheal intubation increased the risk of death by 2.986 times, and the mortality of patients with respiratory failure increased by 4.067 times. Patients with hypoalbuminemia had a 2.408 -fold increased risk of death. The risk of death for patients with MODS was increased by 3.72 times. Patients with a SOFA score greater than 6 had a 3.757-fold increased risk of death compared to other patients (SOFA score $\leq 6$ ). With regard to resistance to carbapenem antibiotics, the risk of death in CRKP infections was 2.9 times higher than that in carbapenem-sensitive Klebsiella pneumoniae (CSKP) infections. 
Table 6

Logistic regression analysis of risk factors of death in patients with $\mathrm{KP}$ bloodstream infections

\section{Characteristics}

Trachea cannula

None

Have

Respiratory failure

None

Have

Hypoalbuminemia

None

Have

MODS

None

Have

SOFA score

$\leq 6$ points

$>6$ points

Characteristics of drug resistance

CRKP

CSKP
OR $(95 \% \mathrm{Cl})$

p

1

$2.986(1.484-6.009) \quad 0.002$

1

$4.067(1.674-9.883) \quad 0.002$

1

$2.408(1.245-4.658)$

0.009

1

$3.720(1.206-11.479)$

0.022

1

$3.757(1.932-7.305)$

$<0.001$

$2.942(1.585-5.461)$

0.001

Abbreviation: KP Klebsiella pneumoniae, $O R$ odds ratio, $C /$ Confidence interval, $C V C$ central venous catheter, MODS multiple organ dysfunction syndrome, SOFA sequential organ failure assessment.

\subsection{Antimicrobial therapy}

Among the 297 patients with Klebsiella pneumoniae BSI, 35 patients were discharged and died before the drug sensitivity was reported, and the other patients used antibiotics for less than 48 hours. 262 patients with Klebsiella pneumoniae BSI who received antimicrobial therapy were divided into groups according to the results of the drug sensitivity test, as shown in Table 7. There were 97 patients with S-KP BSI, with an overall mortality rate of $21.6 \%$. Among them, 81 patients were treated with single antibiotic (83.5\%), and 16 patients were treated with a variety of antibiotics (16.5\%). The combined use of antibiotics did not appear to improve the prognosis. Specifically, carbapenem antibiotics accounted for half of the monotherapy cases, while the remaining cases were treated with cefoperazone sulbactam (24.6\%), 
piperacillin tazobactam (11\%), and cephalosporins (11.3\%). In the combined therapy cases, there were 10 cases in which carbapenem was used and four cases in which tegacycline was used, of which one case was treated with triple antibiotics. There were 87 patients with MDR-KP BSI, and the mortality rate (44.8\%) was higher than that in the sensitive group. Among them, the number of cases treated with monotherapy was relatively less than that of the sensitive group (66.7\%), which was still dominated by carbapenem, with a total of 31 patients. Among them, 18 cases died within 28 days, of which 13 cases $(72.2 \%)$ were resistant to carbapenem, and only one case was resistant to carbapenem. Tegacycline was mainly used for combination treatment $(65.5 \%)$, of which three cases were treated with triple antibiotics. The mortality rate in the single drug treatment group is higher than that in the combination treatment group, which deserves the attention of clinicians. There were 78 patients with XDR-KP BSI, with a mortality rate of $67.9 \%$. Among the patients treated with antibiotic, $55.1 \%$ were treated with a single drug and the case fatality rate was $79 \%$. The case fatality rate of the combined treatment group was $54.2 \%$, of which a combined regimen based on tegacycline accounted for $80 \%$ of the cases. A total of six patients were treated with triple antibiotics, of which one patient died. 
Table 7

Treatment and outcome of the 262 cases of KP bloodstream infection patients

\begin{tabular}{|c|c|c|}
\hline Treatment & n (\%) & Mortality (\%) \\
\hline S-KP & $97(37)$ & $21(21.6)$ \\
\hline Monotherapy & $81(83.5)$ & $15(18.5)$ \\
\hline Carbapenem & $41(50.6)$ & $9(21.9)$ \\
\hline Cefoperazone/sulbactam & $20(24.6)$ & $4(20)$ \\
\hline Piperacillin/tazobactam & $9(11.1)$ & $1(11.1)$ \\
\hline Ceftazidime & $7(8.6)$ & $1(14.2)$ \\
\hline Cefepime & $3(3.7)$ & 0 \\
\hline Cefminox & $1(1.2)$ & 0 \\
\hline Combination therapy & $16(16.5)$ & $6(37.5)$ \\
\hline Tegacycline + Carbapenem & $2(12.5)$ & $1(50)$ \\
\hline Tegacycline + Piperacillin/tazobactam & $1(6.2)$ & $1(100)$ \\
\hline Tegacycline + Carbapenem + Cefoperazone/sulbactam & $1(6.2)$ & 0 \\
\hline Carbapenem + Cefoperazone/sulbactam & $6(37.5)$ & $3(50)$ \\
\hline Carbapenem + Piperacillin/tazobactam & $1(6.2)$ & 0 \\
\hline Carbapenem + Ceftazidime & $2(12.5)$ & $1(50)$ \\
\hline Carbapenem + Cefminox & $1(6.2)$ & 0 \\
\hline Piperacillin/tazobactam + Aminoglycosides & $2(12.5)$ & 0 \\
\hline MDR-KP & $87(33.2)$ & $39(44.8)$ \\
\hline Monotherapy & $58(66.7)$ & $30(51.7)$ \\
\hline Carbapenem & $31(53.4)$ & $18(58)$ \\
\hline Cefoperazone/sulbactam & $10(17.2)$ & $4(40)$ \\
\hline Piperacillin/tazobactam & $9(15.5)$ & $3(33.3)$ \\
\hline Cefepime & $4(6.8)$ & $3(75)$ \\
\hline Ceftazidime & $2(3.4)$ & $1(50)$ \\
\hline Cefotiam & $2(3.4)$ & $1(50)$ \\
\hline Combination therapy & $29(33.3)$ & $9(31)$ \\
\hline
\end{tabular}




\begin{tabular}{|c|c|c|}
\hline Treatment & $\mathrm{n}(\%)$ & Mortality (\%) \\
\hline Tegacycline + Carbapenem & $7(24.1)$ & $3(42.8)$ \\
\hline Tegacycline + Polymyxin B & $1(3.4)$ & 0 \\
\hline Tegacycline + Piperacillin/tazobactam & $2(6.8)$ & $1(50)$ \\
\hline Tegacycline + Aminoglycosides & $5(17.2)$ & $1(20)$ \\
\hline Tegacycline + Cefoperazone/sulbactam & $1(3.4)$ & $1(100)$ \\
\hline Tegacycline + Carbapenem + Cefoperazone/sulbactam & $2(6.8)$ & 0 \\
\hline Tegacycline + Carbapenem + Aminoglycosides & $1(3.4)$ & 0 \\
\hline Carbapenem + Polymyxin B & $1(3.4)$ & $1(100)$ \\
\hline Treatment & $\mathrm{n}(\%)$ & Mortality (\%) \\
\hline Carbapenem + Piperacillin/tazobactam & $1(3.4)$ & 0 \\
\hline Carbapenem + Cefoperazone/sulbactam & $4(13.8)$ & $1(25)$ \\
\hline Carbapenem + Ceftazidime & $2(6.8)$ & $1(50)$ \\
\hline Piperacillin/tazobactam + Aminoglycosides & $2(6.8)$ & 0 \\
\hline XDR-KP & $78(29.7)$ & $53(67.9)$ \\
\hline Monotherapy & $43(55.1)$ & $34(79)$ \\
\hline Carbapenem & $25(58.1)$ & $22(88)$ \\
\hline Cefoperazone/sulbactam & $10(23.2)$ & $9(90)$ \\
\hline Piperacillin/tazobactam & $5(11.6)$ & $2(40)$ \\
\hline Cefepime & $1(2.3)$ & $1(100)$ \\
\hline Ceftazidime & $2(4.6)$ & 0 \\
\hline Combination therapy & $35(44.9)$ & $19(54.2)$ \\
\hline Tegacycline + Carbapenem & $11(31.4)$ & 7 (63.6) \\
\hline Tegacycline + Polymyxin B & $1(2.8)$ & $1(100)$ \\
\hline Tegacycline + Piperacillin/tazobactam & $4(11.4)$ & $2(50)$ \\
\hline Tegacycline + Aminoglycosides & $3(8.5)$ & $2(66.7)$ \\
\hline Tegacycline + Cefoperazone/sulbactam & $4(11.4)$ & $3(75)$ \\
\hline Tegacycline + Carbapenem + Cefoperazone/sulbactam & $3(8.5)$ & 0 \\
\hline
\end{tabular}

Continued Table 7. Treatment and outcome of the 262 cases of KP bloodstream infection patients 


\begin{tabular}{|lll|}
\hline Treatment & $\mathbf{n}(\%)$ & Mortality (\%) \\
\hline Tegacycline+ Carbapenem + Aminoglycosides & $1(2.8)$ & 0 \\
\hline Tegacycline + Carbapenem + Polymyxin B & $1(2.8)$ & $1(100)$ \\
\hline Carbapenem + Cefoperazone/sulbactam & $4(11.4)$ & $3(75)$ \\
\hline Carbapenem + Aminoglycosides & $1(2.8)$ & 0 \\
\hline Carbapenem + Cefepime & $1(2.8)$ & 0 \\
\hline Carbapenem + Aminoglycosides + Cefoperazone/sulbactam & $1(2.8)$ & 0 \\
\hline $\begin{array}{l}\text { Abbreviation: } n \text { sample size, S-KP sensitive Klebsiella pneumoniae, MDR-KP multidrug resistant } \\
\text { Klebsiella pneumoniae, XDR-KP extensively drug resistant Klebsiella pneumoniae. }\end{array}$ & \\
\hline Continued Table 7. Treatment and outcome of the 262 cases of KP bloodstream infection patients \\
\hline
\end{tabular}

\section{Discussion}

Since the beginning of this century, Klebsiella pneumoniae has been prevalent all over the world, and with increasing incidence. It has gained the attention of clinical workers: on the one hand, the increase in the detection rate of drug-resistant bacteria has made it more difficult for clinicians to choose antibiotics; on the other hand, drug-resistant Klebsiella pneumoniae infections have a high mortality rate(2). It has been recommended that a combination of antibiotics be used to treat Klebsiella pneumoniae BSI, but there is still no further clinical evidence. Therefore, we collected the clinical data of patients with Klebsiella pneumoniae bacteremia and statistically analyzed the risk factors for drug-resistant bacterial infection, the risk factors for death, and the mortality of patients with different drug-resistant Klebsiella pneumoniae. The treatment plan was evaluated according to patient prognosis to improve the alertness of clinicians with regard to these cases and to provide guidance for the prevention of drug-resistant bacterial infections and reasonable treatment.

We collected and analyzed the clinical data of patients with Klebsiella pneumoniae BSI during the past five years. Of the 297 patients, S-KP accounted for only $38.4 \%$ of the total number of cases. Reviewing data on Klebsiella pneumoniae isolated from blood samples released in Greece, its resistance to carbapenem antibiotics ranged from less than $1 \%$ in 2001 to $30-60 \%$ in 2008. In 2006, researchers found that the reason for carbapenem resistance of this batch of CRKP was the presence of the bla $\mathrm{VIIM}_{-1}$ gene $(28,29)$. In 2008, a number of studies found the bla KPC-2 $_{2}$ gene present in CRKP isolates and, at one time, there was an epidemic in two separate hospitals(30,31). Subsequently, CRKP was detected in the United States, Italy, Israel, and other countries around the world, one after another. In this study, we did not explore the molecular epidemiology, but put more emphasis on the clinician perspective. We compared the clinical characteristics of Klebsiella pneumoniae patients with different drug resistance and found that sex and age were not related to susceptibility to drug-resistant bacteria. Patients with recent ICU hospitalization history, recent surgical history, recent antibiotic use history, mechanical ventilation history, 
and hospitalization more than two weeks before the BSI diagnosis were more likely to be infected with drug-resistant bacteria. It is suggested that clinicians be more vigilant against the possibility of drugresistant bacterial infections when facing the clinical manifestations of BSIs in these kinds of patients, such as fever, increased procalcitonin (PCT), or even shock. At the same time, several other possible risk factors should not be ruled out, such as patients with a recent history of invasive surgery, patients in the ICU ward, patients with diabetes, and patients with severe clinical symptoms. Mantzarlis et al. found that critically ill patients who received carbapenem or polymyxin antibiotics, patients who received mechanical ventilation, and patients who underwent invasive surgery were more likely to develop CRKP infection(32). However, all the patients included were ICU patients, and the samples included blood and airway secretions, as well as other infectious secretions. Freeman et al. found that patients with a history of ICU hospitalization and transplantation, including hematopoietic stem cell and solid organ transplant, had a higher risk of Extended-Spectrum $\beta$-Lactamases Klebsiella pneumoniae (ESBL-KP) infection(33). However, fewer cases were included in this study, and there was no age limit. At the same time, patients who do not have ESBL-KP may also have carbapenem or even a variety of antibiotic-resistant bacteria. In this study, based on the results of the drug sensitivity test that clinicians are typically most concerned about, we analyzed the epidemiological characteristics, high risk factors, and prognosis for drug-resistant bacterial infections.

In this study, the 28-day all-cause mortality rate of the included patients was $42.8 \%$. Patients were divided into groups according to the results of the different drug sensitivity tests. The results showed that the mortality rate of patients with S-KP bacteremia was $21.6 \%$. The mortality rate of patients with MDR-KP bacteremia was $44.8 \%$. And the mortality rate of patients with XDR-KP bacteremia was $67.9 \%$. Studies by Hoxha et al. found that the 30-day attributable mortality rate of CRKP patients was $41 \%$, and the risk of death was three times higher than that of CSKP patients(34). However, the inclusion population included all patients with Klebsiella pneumoniae infection, not limited to BSIs. Studies by Borer et al. found that the crude and attributable mortality rates of patients with drug-resistant Klebsiella pneumoniae were $71.9 \%$ and $50 \%$, respectively(35). It was also found that the mortality rate of patients with MODS was $86.9 \%$, and that of patients with septic shock was $100 \%$, which was significantly higher than that of patients with simple sepsis. Nearly $25 \%$ of the patients in the study conducted by Borer et al. were from nursing homes and, thus, pertained more to elderly patients. Only adult patients with clinically diagnosed BSI and only Klebsiella pneumoniae blood cultures were included in our study. The mortality data obtained are higher than that in previous studies. On the one hand, because of the large number of critically ill patients, a total of $38 \%$ of the patients had a history of living in ICU before hospitalization, and a total of $61.6 \%$ of the patients were admitted to ICU during hospitalization. On the other hand, because the outcome index uses a survival condition of 28 days after the BSI diagnosis, this study pertains to allcause mortality rather than hospital mortality. Unlike other studies that include only CRKP or XDR-KP, this trial includes information on all patients with Klebsiella pneumoniae BSI with different drug sensitivity results.

We analyzed the patients with different clinical prognoses. Through univariate and multivariate analysis, it was clear that tracheal intubation, respiratory failure, hypoalbuminemia, MODS, a SOFA score greater 
than 6 points, and CRKP were independent risk factors for death within 28 days after diagnosis of Klebsiella pneumoniae infection. Additionally, patients with poor prognosis were more likely to get worse during the course of treatment, such as MODS, acute kidney injury (AKI), mechanical ventilation, and septic shock. The risk factors explored in this study were 28-day risk factors for death in all patients clinically and bacteriologically diagnosed with Klebsiella pneumoniae BSI. Mantzarlis et al. found that older patients and immunodeficient patients were independent risk factors for death in patients with CRKP infection in the ICU(36). Recent studies in southwest China have found that XDR-KP patients with solid tumors and septic shock are independent risk factors for death within 28 days. However, sputum was the main sample and only $13 \%$ of the samples were blood. At the same time, the positive culture results of the samples were not combined with the corresponding clinical symptoms. Falcone et al. showed that colistin resistance and intra-abdominal infection were independent risk factors for death in patients with CRKP septic shock(37). Machuca et al. found that septic shock and admission to the ICU are independent risk factors for 30-day death in patients with CRKP, and combined anti-infective therapy can reduce the mortality of septic shock patients with CRKP bacteremia(38). The detection rate of drugresistant bacteria is increasing day by day, and the 2.9 times higher mortality rate of patients with CRKP infection versus CSKP patients highlights the importance of developing pre-control.

Compared to different treatment schemes, anti-infective combination therapy in patients with S-KP bacteremia did not demonstrate a significant benefit; whereas, monotherapy had a relatively lower cost and greater benefit. Although patients with MDR-KP bacteremia had a lower risk of monotherapy, more patients died in 28 days, and the two antibiotic regimens dominated by tigacycline or carbapenem appeared more frequently. The mortality rate of patients with XDR-KP bacteremia is very high. We found that the 28-day mortality rate of combined therapy was significantly lower than that of monotherapy. It is recommended to avoid anti-infective therapy with single antibiotics in the case of drug-resistant bacterial infections. For patients at high-risk for drug-resistant bacterial infection, if the possibility of Gramnegative bacteremia is considered, whether there is clinical benefit for combination anti-infective treatment to treat Klebsiella pneumoniae infection still needs to be confirmed by further clinical studies.

Our experiment has some limitations. First of all, we only counted the all-cause mortality of patients, but we included only patients with clear Klebsiella pneumoniae at the time of selection, excluding patients who died or were discharged within 24 hours after admission. Second, our sample size is small and retrospective observational studies were conducted. A larger sample is necessary for further randomized controlled trials, but taking into account the changes in drug use and the drug resistance spectrum over time, the patient data from the last five years were selected and no further review was conducted. Third, we did not compare the prognosis of treatment with binary and triple antibiotics. This was due to the fewer number of cases for combined use of three kinds of antibiotics. Thus, the reference value for comparison is small. Finally, and most importantly, our study does not represent the current epidemiological situation in China, let alone other countries. The specific distribution of drug resistance and the corresponding treatment plans still need to be further developed according to the local epidemiological characteristics. 


\section{Conclusion}

In short, our study found that the detection rate of drug-resistant bacteria was high in patients with Klebsiella pneumoniae BSI. Patients with a recent ICU hospitalization history, recent surgical history, recent antibiotic use history, and mechanical ventilation history were more likely to be infected with drugresistant bacteria, and the susceptibility rate of the drug-resistant bacteria in patients who were hospitalized for more than two weeks before BSI was 4.34 times higher than that in patients with less than one week of hospitalization. The prognosis for drug-resistant bacterial infection is poor, especially XDR-KP infection, and the mortality rate is high. Combined treatment of more than two kinds of antibiotics is recommended in XDR-KP bacteremia patients.

\section{Abbreviations}

BSI: Bloodstream infection; MODS: Multiple organ dysfunction syndrome; CRKP: Carbapenem-resistant Klebsiella pneumoniae; ICU: Intensive care unit; XDR: Extensively drug- resistant; XDR-KP: Extensively drug-resistant Klebsiella pneumoniae; CDC: Centers for Disease Control and Prevention; CLABI: Central Line-Associated Bloodstream Infection; SCCM: Society of Critical Care Medicine; ESCIM: European Society of Intensive Care Medicine; MDR: Multidrug resistance; PDR: Pandrug resistance; EUCAST: European Committee on Antimicrobial Susceptibility Testing; CKD: Chronic kidney disease; APACHE: Acute Physiology and Chronic Health Evaluation; SOFA: Sequential Organ Failure Assessment; CVC: Central venous catheter; SD: Standard deviation; IQR: Interquartile range; OR: Odds ratio; Cl: Confidence interval; S-KP: Sensitive Klebsiella pneumoniae; MDR-KP: Multidrug resistant Klebsiella pneumoniae; CSKP: Carbapenem-sensitive Klebsiella pneumoniae; ESBL-KP: Extended-Spectrum $\beta$-Lactamases Klebsiella pneumoniae.

\section{Declarations}

Ethics approval and consent to participate

Our study has been approved by the Scientific Research and Clinical Trial Ethics Committee of the First Affiliated Hospital of Zhengzhou University (Code 2020-KY-087).

Consent for publication

Not applicable.

Availability of data and materials

All data generated or analyzed during this study are included in this published article and its supplementary information files.

Competing interests 
The authors declare that they have no competing interests.

\section{Funding}

This study was supported by the Overseas Research and Training Project of Talents of Health and Health Science and Technology in Henan Province in 2019 (Grant No. HWYX2019020), the Scientific and Technological Innovation Leaders in Central Plains (Grant No. 194200510017), Provincial Ministry CoConstruction Project from the Medical Scientific and Technological Research Program of Henan Province (Grant No. SBGJ2018020), the "51282" Project Leaders of Scientific and Technological Innovative Talents from Health and Family Planning Commission in Henan Province (2016-32), and Science and Technology People-Benefit Project of Zheng Zhou (2019KJHM0001).

\section{Authors' contributions}

SGZ and ZYY conceived and designed the study. LMS, LTS and JLX participated in the collection of case data. SGZ and TWS conducted statistical analyses, and wrote the paper. All authors read and approved the final manuscript.

\section{Acknowledgements}

Not applicable.

\section{References}

1. Willyard C. The drug-resistant bacteria that pose the greatest health threats. Nature 2017;543:15.

2. Rodriguez-Bano J, Gutierrez-Gutierrez B, Machuca I, et al. Treatment of Infections Caused by Extended-Spectrum-Beta-Lactamase-, AmpC-, and Carbapenemase-Producing Enterobacteriaceae. Clin Microbiol Rev 2018;31.

3. Denkel LA, Maechler F, Schwab F, et al. Infections caused by extended-spectrum beta-lactamaseproducing Enterobacterales after rectal colonization with ESBL-producing Escherichia coli or Klebsiella pneumoniae. Clin Microbiol Infect 2019.

4. Sequeira RP, McDonald JAK, Marchesi JR, et al. Commensal Bacteroidetes protect against Klebsiella pneumoniae colonization and transmission through IL-36 signalling.1.

5. Russo TA, Marr CM. Hypervirulent Klebsiella pneumoniae. Clin Microbiol Rev 2019;32.

6. Selden R, Lee S, Wang WL, et al. Nosocomial klebsiella infections: intestinal colonization as a reservoir.1.

7. Dorman MJ, Short FL. Genome watch: Klebsiella pneumoniae: when a colonizer turns bad.1.

8. Du B, Long Y, Liu H, et al. Extended-spectrum beta-lactamase-producing Escherichia coli and Klebsiella pneumoniae bloodstream infection: risk factors and clinical outcome.1.

9. Garnacho-Montero J, Aldabo-Pallas T, Palomar-Martinez M, et al. Risk factors and prognosis of catheter-related bloodstream infection in critically ill patients: a multicenter study.1. 
10. Laupland KB, Zygun DA, Doig CJ, et al. One-year mortality of bloodstream infection-associated sepsis and septic shock among patients presenting to a regional critical care system.1.

11. Kim PW, Perl TM, Keelaghan EF, et al. Risk of mortality with a bloodstream infection is higher in the less severely ill at admission.1.

12. Rahal JJ. The role of carbapenems in initial therapy for serious Gram-negative infections. [Review] [55 refs]. 1 .

13. Potter RF, D'Souza AW, Dantas G. The rapid spread of carbapenem-resistant Enterobacteriaceae. [Review].1.

14. MacKenzie FM, Forbes KJ, Dorai-John T, et al. Emergence of a carbapenem-resistant Klebsiella pneumoniae.1.

15. Capone A, Giannella M, Fortini D, et al. High rate of colistin resistance among patients with carbapenem-resistant Klebsiella pneumoniae infection accounts for an excess of mortality. 1.

16. Daikos GL, Tsaousi S, Tzouvelekis LS, et al. Carbapenemase-producing Klebsiella pneumoniae bloodstream infections: lowering mortality by antibiotic combination schemes and the role of carbapenems. 1.

17. Lee NY, Tsai CS, Syue LS, et al. Treatment Outcome of Bacteremia Due to Non-Carbapenemaseproducing Carbapenem-Resistant Klebsiella pneumoniae Bacteremia: Role of Carbapenem Combination Therapy.1.

18. Tsala M, Vourli S, Georgiou PC, et al. Triple combination of meropenem, colistin and tigecycline was bactericidal in a dynamic model despite mere additive interactions in chequerboard assays against carbapenemase-producing Klebsiella pneumoniae isolates. 1.

19. Schwaber MJ, Carmeli Y. Carbapenem-resistant Enterobacteriaceae: a potential threat.1.

20. Gomez-Simmonds A, Nelson B, Eiras DP, et al. Combination Regimens for Treatment of CarbapenemResistant Klebsiella pneumoniae Bloodstream Infections.1.

21. Ben-David D, Kordevani R, Keller N, et al. Outcome of carbapenem resistant Klebsiella pneumoniae bloodstream infections. 1.

22. Lee Y, Kim YA, Kim D, et al. Risk factors of community-onset extended-spectrum beta-lactamaseproducing Klebsiella pneumoniae bacteraemia in South Korea using national health insurance claims data. Int J Antimicrob Agents 2019;54:723-7.

23. Giannella M, Pascale R, Gutierrez-Gutierrez B, et al. The use of predictive scores in the management of patients with carbapenem-resistant Klebsiella pneumoniae infection. Expert Rev Anti Infect Ther 2019;17:265-73.

24. Gutierrez-Gutierrez B, Salamanca E, de Cueto M, et al. Effect of appropriate combination therapy on mortality of patients with bloodstream infections due to carbapenemase-producing Enterobacteriaceae (INCREMENT): a retrospective cohort study. Lancet Infect Dis 2017;17:726-34.

25. Doi Y. Treatment Options for Carbapenem-resistant Gram-negative Bacterial Infections. Clin Infect Dis 2019;69:S565-s75. 
26. Singer M, Deutschman CS, Seymour CW, et al. The Third International Consensus Definitions for Sepsis and Septic Shock (Sepsis-3). Jama 2016;315:801-10.

27. Magiorakos AP, Srinivasan A, Carey RB, et al. Multidrug-resistant, extensively drug-resistant and pandrug-resistant bacteria: an international expert proposal for interim standard definitions for acquired resistance. Clin Microbiol Infect 2012;18:268-81.

28. Souli M, Kontopidou FV, Papadomichelakis E, et al. Clinical experience of serious infections caused by Enterobacteriaceae producing VIM-1 metallo-beta-lactamase in a Greek University Hospital. Clin Infect Dis 2008;46:847-54.

29. Vatopoulos A. High rates of metallo-beta-lactamase-producing Klebsiella pneumoniae in Greece-a review of the current evidence. Euro Surveill 2008;13.

30. Maltezou HC, Giakkoupi P, Maragos A, et al. Outbreak of infections due to KPC-2-producing Klebsiella pneumoniae in a hospital in Crete (Greece). J Infect 2009;58:213-9.

31. Pournaras S, Protonotariou E, Voulgari E, et al. Clonal spread of KPC-2 carbapenemase-producing Klebsiella pneumoniae strains in Greece. J Antimicrob Chemother 2009;64:348-52.

32. Mantzarlis K, Makris D, Manoulakas E, et al. Risk factors for the first episode of Klebsiella pneumoniae resistant to carbapenems infection in critically ill patients: a prospective study. Biomed Res Int 2013;2013:850547.

33. Freeman JT, Rubin J, McAuliffe GN, et al. Differences in risk-factor profiles between patients with ESBL-producing Escherichia coli and Klebsiella pneumoniae: a multicentre case-case comparison study.1.

34. Hoxha A, Karki T, Giambi C, et al. Attributable mortality of carbapenem-resistant Klebsiella pneumoniae infections in a prospective matched cohort study in Italy, 2012-2013. J Hosp Infect 2016;92:61-6.

35. Borer A, Saidel-Odes L, Riesenberg K, et al. Attributable mortality rate for carbapenem-resistant Klebsiella pneumoniae bacteremia. Infect Control Hosp Epidemiol 2009;30:972-6.

36. Tian X, Huang C, Ye X, et al. Molecular Epidemiology of and Risk Factors for Extensively DrugResistant Klebsiella pneumoniae Infections in Southwestern China: A Retrospective Study. Front Pharmacol 2019;10:1307.

37. Falcone M, Russo A, lacovelli A, et al. Predictors of outcome in ICU patients with septic shock caused by Klebsiella pneumoniae carbapenemase-producing K. pneumoniae. Clin Microbiol Infect 2016;22:444-50.

38. Machuca I, Gutierrez-Gutierrez B, Rivera-Espinar F, et al. External validation of the INCREMENT-CPE mortality score in a carbapenem-resistant Klebsiella pneumoniae bacteraemia cohort: the prognostic significance of colistin resistance. Int J Antimicrob Agents 2019;54:442-8. 